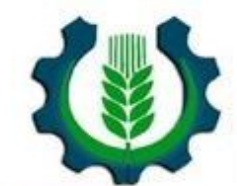

BIOENG

\title{
GINÁSTICA LABORAL EM CAVALOS TERAPEUTAS MELHORA A QUALIDADE DO MOVIMENTO TRIDIMENSIONAL DO TROTE
}

${ }^{1}$ UNESP - Universidade Estadual Paulista, Faculdade de Ciências Agrarias e Tecnológicas, Dracena, SP, Brasil

${ }^{2}$ UNESP - Universidade Estadual Paulista, Faculdade de Medicina Veterinária e Zootecnia, Botucatu, SP, Brasil

Article history: Received 12 March 2019; Received in revised form 22 May 2019; Accepted 27 May 2019; Available online 24 June 2019.

\section{RESUMO}

Objetivou-se determinar se cavalos terapeutas submetidos a ginástica laboral desenvolvem melhor qualidade do movimento tridimensional ao trote, avaliado por meio das variáveis biomecânicas como, cadência, regularidade e simetria. Foram utilizados vinte cavalos terapeutas, mestiços, com idade média de $19 \pm 3,5$ anos e peso vivo médio de $440 \pm 50 \mathrm{~kg}$. Os tratamentos consistiram em um total de cinco grupos experimentais, compostos por grupo controle (sem realização de exercícios) e de quatro grupos testes de cavalos submetidos a ginástica laboral, com frequências semanais crescentes de realização, variando de uma à quatro vezes/semana. A ginástica laboral foi conduzida durante período de três meses. $\mathrm{O}$ delineamento utilizado foi inteiramente casualizado, resultando em quatro repetições por tratamento. Foram mensuradas as variáveis biomecânicas, referentes a coordenação do andamento ao trote como, cadência da passada (CAD), regularidade da passada (REG) e simetria da passada (SIM), para determinar a qualidade do movimento tridimensional, por meio do acelerômetro Equimetrix. Foi verificada ausência de efeito significativo para o ganho na CAD ao trote $(\mathrm{P}>0,05)$. Contudo, foi observado melhora nos ganhos da REG e SIM $(\mathrm{P}<0,05)$, em que os melhores resultados foram encontrados aos cavalos submetidos a ginástica laboral realizada de três à quatro vezes/semana, com valores de 15,4 e 16,0 (/200) para REG e de 14,9 e 20,6 (\%) para SIM, respectivamente. Concluiu-se que a realização de ginástica laboral em cavalos terapeutas, a partir de três sessões semanais, melhora o movimento tridimensional do trote, devido ao ganho na regularidade e simetria, mantendo-se a mesma cadência de passada.

Palavras-chave: biomecânica, cinemática, equoterapia, treinamento funcional

\section{GYMNASTIC TRAINING IN THERAPY HORSES IMPROVES QUALITY OF THE TRIDIMENSIONAL MOVEMENT OF TROT}

\begin{abstract}
The objective of this study was to determine if therapy horses submitted to gymnastic training developed a better quality of three-dimensional movement of trot, evaluated by means of biomechanical variables as cadence, regularity and symmetry. Twenty therapy horses were used with a mean age of $19 \pm 3.5$ years and mean live weight of $440 \pm 50 \mathrm{~kg}$. The treatments consisted of a total of five experimental groups, consisting of a control group (without
\end{abstract}

\footnotetext{
*katia.oliveira@unesp.br
} 
exercise) and four test groups of horses submitted to gymnastic training, with weekly frequencies increasing, ranging from one to four times a week. Gymnastic training was performed during a three-month period. The design was completely randomized, resulting in four replications per treatment. The biomechanical variables related to the coordination of the gait were analyzed, such as, cadence of the stride (CAD), regularity of the stride (REG) and symmetry of the stride (SYM), to determine the quality of the three-dimensional movement, using the Equimetrix accelerometer. There was no significant effect for trotting CAD gain $(\mathrm{P}>$ 0.05). However, an improvement in the gains of REG and SYM was observed $(P<0.05)$, in which the best results were found in horses submitted to gymnastic training performed three to four times a week, with values of 15.4 and 16.0 (/ 200) for REG and of 14.9 and 20.6 (\%) for SYM, respectively. It was concluded that the performance of gymnastic training in therapy horses, from three sessions per week, improves the three-dimensional movement of the trot, due to the gain in regularity and symmetry, with the same cadence of the stride.

Keywords: biomechanics, kinematics, equine therapy, functional training

\section{INTRODUÇÃO}

A equoterapia é um procedimento terapêutico que visa a utilização do cavalo como instrumento de tratamento aos praticantes portadores de deficiências ou de necessidades especiais. A aplicação cinesioterapêutica desta prática fundamenta-se no movimento tridimensional realizado pelo animal, durante seu deslocamento, que movimenta a pélvis do cavaleiro em padrão similar ao caminhar humano (ENCHEFF et al., 2012). Sendo assim, a terapia possibilita ganhos efetivos nos domínios físicos, psicológicos, cognitivos e sociais, pela combinação única do cavalo, andamento e ambiente não clínico, que produz extraordinário esforço em todos os sistemas do corpo do paciente (HAKANSON et al., 2009). Devido a estes benefícios à saúde do paciente, a terapia montada tem recebido alta demanda e procura crescente da população, provocando nos centros de equoterapia lentidão nos agendamentos às sessões, bem como a utilização de cavalos em más condições físicas (SILVA, 2012).

Para o cavalo de terapia realizar com eficiência a sua função como ferramenta reabilitadora, o movimento tridimensional, ou seja, a qualidade do andamento é de suma importância (SVOBODA et al., 2011). De acordo com ROSA (2006), o cavalo de terapia deve ter extremo potencial de movimento nos andamentos, para que o praticante receba tratamento de qualidade, acelerando o processo terapêutico e reabilitativo. Assim, cavalos contendo irregularidades em seus andamentos, mesmo não sendo perceptíveis ou visíveis, apresentam decréscimos nas características biomecânicas como regularidade, simetria e cadência, prejudicando o movimento tridimensional (LÓPEZ-SANROMÁN et al., 2012).

A integridade biomecânica nos equinos é alcançada por meio de uma rotina de exercícios, realizados nos cavalos, para que ocorra manutenção de sua condição física, prevenindo à contínua e gradual perda da qualidade dos andamentos (STUBBS et al., 2011). Sendo assim, recente estudo avaliou o efeito da ginástica laboral em cavalos de terapia sobre a cinemática do passo e desenvolvimento muscular (OLIVEIRA et al., 2015). Esta atividade funcional foi composta por exercícios de mobilização dinâmica, de fortalecimento muscular ao abdômen e de estabilização da pélvis, realizada três vezes por semana, durante dois meses. Comprovou-se que a rotina de exercícios resultou em melhor qualidade do passo $(\mathrm{P}<0,05)$ e hipertrofia do musculus multifidus, significativamente, em equinos terapeutas. 
Vale ressaltar que, a temática sobre a importância da ginástica laboral em cavalos terapeutas deve ser difundida para garantir o bem-estar aos equinos, bem como para assegurar tratamento terapêutico de qualidade aos usuários. Portanto, melhorias na qualidade do atendimento em terapia montada devem começar pela principal ferramenta que

\section{MATERIAL E MÉTODOS}

A presente pesquisa foi certificada pela "Comissão de Ética em Uso de Animais", sob no 20/2018.R1, estando de acordo com os princípios éticos de experimentação animal. Foram utilizados vinte cavalos terapeutas e mestiços. $\mathrm{O}$ critério para inclusão de cavalos ao estudo foi nenhuma claudicação visível, sem sinais ou sintomas compatíveis com lesões musculoesqueléticas, habituados às sessões de equoterapia por três anos e trabalhando regularmente. Esta seleção resultou na utilização de equinos mestiços, com idade

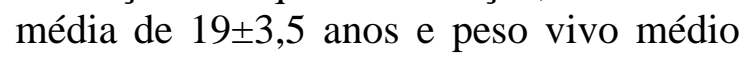
de $440 \pm 50 \mathrm{~kg}$.

Os tratamentos consistiram em um total de cinco grupos experimentais, compostos por grupo controle (sem realização de exercícios) e de quatro grupos testes de cavalos submetidos a ginástica laboral, com frequências semanais crescentes de realização, variando de uma à quatro vezes/semana. $\mathrm{O}$ delineamento utilizado foi inteiramente casualizado, resultando em quatro repetições por tratamento. A ginástica laboral foi desenvolvida em pesquisa anterior, conforme OLIVEIRA et al., (2015), consistindo de exercícios de mobilização dinâmica (STUBBS et al., 2011), de fortalecimento muscular ao abdômen (HIGGINS, 2009) e de estabilização da pélvis (STUBBS \& CLAYTON, 2008). Em período préexperimental de um mês os cavalos dos grupos testes foram habituados aos exercícios funcionais da ginástica laboral. Após esta fase, em período experimental com duração de três meses, a ginástica foi proporciona a reabilitação ao paciente, ou seja, o cavalo. Neste sentido, objetivou-se determinar se cavalos terapeutas submetidos a ginástica laboral desenvolvem melhor qualidade do movimento tridimensional ao trote, avaliado por meio das variáveis biomecânicas como, cadência, regularidade e simetria.

realizada nos cavalos à frequência de uma à quatro vezes/semana, conforme designação do tratamento.

A ginástica laboral foi composta por exercícios de mobilização dinâmica e de fortalecimento muscular. A série de exercícios de mobilização dinâmica foi constituída por três exercícios de flexão cervical longitudinal, cabeça entre os cascos torácicos, cabeça entre carpos e cabeça no manúbrio, (Figura 1), um exercício de extensão cervical (Figura 2) e três exercícios de flexão cervical lateral (direita e esquerda), cabeça na espádua, cabeça na patela e cabeça no jarrete, (Figura 3), totalizando em dez mobilizações. Cada exercício de mobilização foi repetido por cinco vezes, para cada sessão de exercícios, mantidos por 5 segundos, e feito com auxílio de um petisco, para conduzir os cavalos às posições desejadas. $\mathrm{O}$ fortalecimento abdominal foi composto pelos exercícios de recuo (Figura 4), spinning (Figura 4) e flexão bilateral da pélvis (Figura 5) e, utilizando-se da passagem sobre obstáculo ao passo (Figura 4), para fortalecer os músculos estabilizadores da pélvis. Os exercícios de fortalecimento muscular seguiram a descrição detalhada contida no trabalho de HIGGINS (2009). Assim, o recuo foi feito em uma única série, de dez passos consecutivos em linha reta, o spinning foi realizado com auxílio de um tambor, perfazendo três repetições para cada lado por sessão, a inclinação da pélvis foi feita, gentilmente, por meio de ponto reflexo localizado entre o bíceps femoris e semitendinosus (cinco repetições por 
sessão) e a passagem ao passo pelo obstáculo, foi utilizado cavalete erguido a
$40 \mathrm{~cm}$ de altura, durante dez minutos, nos sentidos horários e anti-horários.

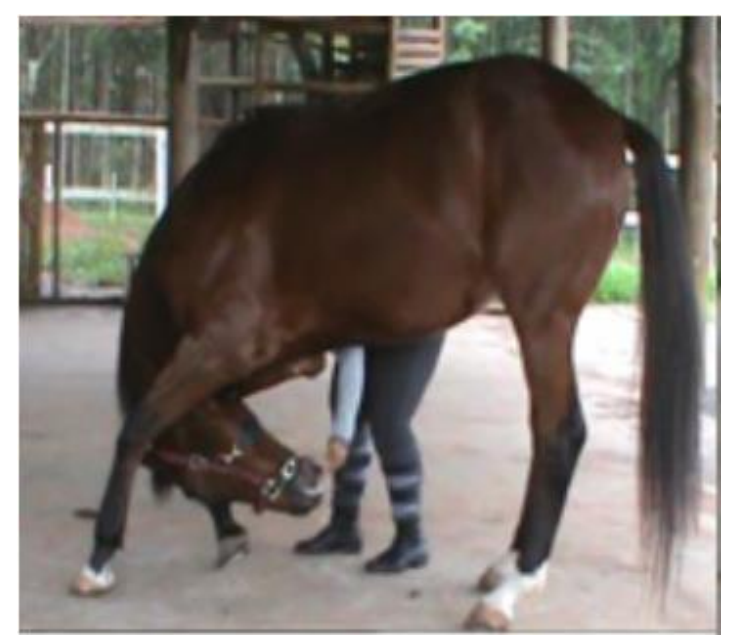

Figura 1. Flexão cervical longitudinal, cabeça entre carpos

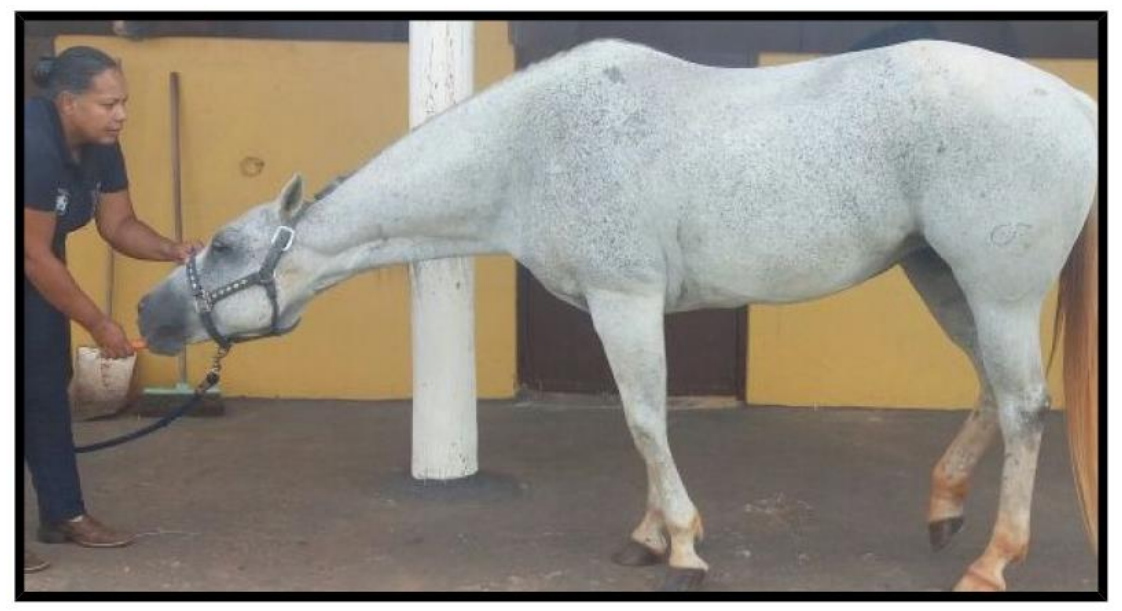

Figura 2. Extensão cervical

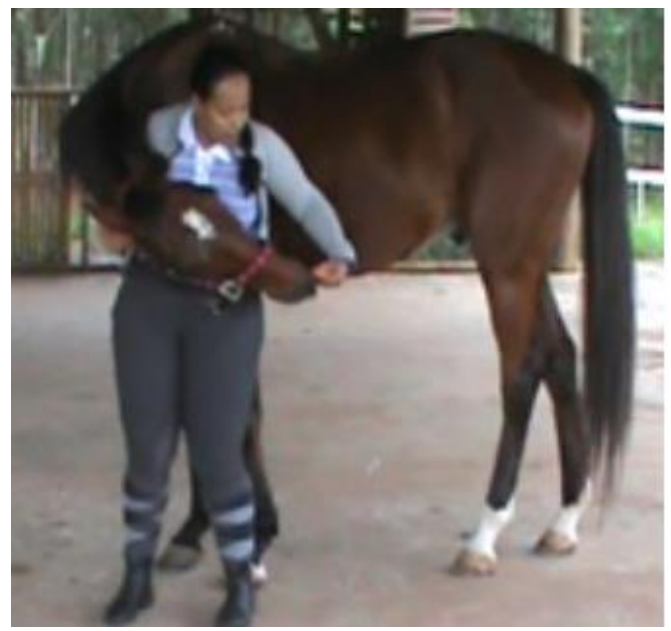

Figura 3. Flexão cervical lateral, cabeça na espádua 


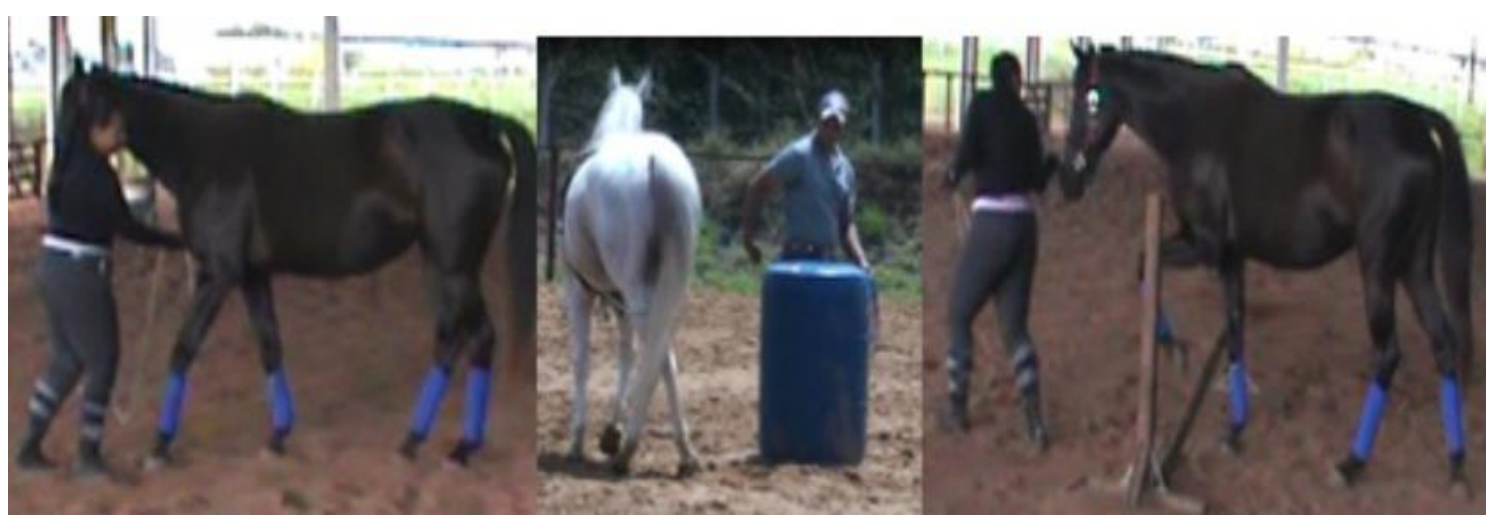

Figura 4. Da esquerda para direita, ilustrações do recuo, spnning e passagem pelo obstáculo

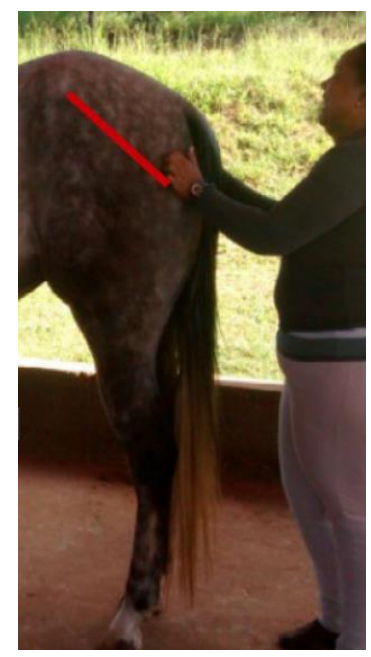

Figura 5. Flexão bilateral da pélvis

Foram mensuradas as variáveis biomecânicas, referentes a coordenação do andamento ao trote como, cadência da passada (CAD), regularidade da passada (REG) e simetria da passada (SIM), para determinar a qualidade do movimento tridimensional, por meio do acelerômetro Equimetrix. A variável CAD representou a frequência de passada, que foi definida como o número de passadas por unidade de tempo e expressa em número de passadas/seg. A REG mediu o padrão de similaridade entre uma passada em relação a outra (entre passadas) em um determinado espaço de tempo (/200). A SIM mensurou o grau de similaridade entre uma meia passada em relação outra, ou seja, dentro da mesma passada (em \%).

O sistema de análise do andamento do Equimetrix foi composto por um transdutor de aceleração conectado a um pequeno gravador de dados. $\mathrm{O}$ transdutor foi constituído por três acelerômetros ortogonais, que medem a aceleração do tórax ao longo dos eixos dorso-ventral, médio-lateral e longitudinal do cavalo. Os dados foram coletados a uma taxa de frequência de $50 \mathrm{Hertz}$ por $10 \mathrm{~min}$. O equimetrix foi fixado entre os músculos peitorais ascendentes direto e esquerdo, localizando-se a frente e cerca de $60 \mathrm{~cm}$ abaixo do centro de gravidade do cavalo (BARREY \& GALLOUX, 1997). Ainda, após as gravações dos dados, foi utilizado o software do próprio programa para obtenção dos valores das variáveis biomecânicas (BARREY et al., 2002).

As variáveis biomecânicas mensuradas foram obtidas por meio de duas avaliações, em período experimental, sendo a primeira realizada antes de se iniciar os exercícios (dia 0), e a segunda mensuração obtida após três meses de atividade física (dia 90). Para realização 
das avaliações foi utilizada uma pista de areia. Previamente as avaliações, os cavalos foram aquecidos por 15 minutos, nos andamentos ao passo e trote, guiados pelo cabresto, para depois serem conduzidos à pista de areia para mensuração (JANURA et al., 2010). Foram realizadas avaliações no trote à $8 \mathrm{~m} / \mathrm{s}$, monitorado por fotocélula, de acordo com JANURA et al. (2010). Desta forma, o deslocamento dos cavalos foi gravado por no mínimo 25 segundos mediante uso de acelerômetro (BARREY et al. 2002).

\section{RESULTADOS E DISCUSSÃO}

Na tabela 1 estão apresentados os valores médios das variáveis biomecânicas no andamento ao trote, de cavalos submetidos a frequências semanais crescentes de execução da ginástica laboral. Após análise dos resultados foi verificada ausência de efeito significativo para o
Os dados biomecânicos de qualidade do movimento tridimensional estão apresentados como média, tendo como medida de dispersão o desvio padrão ( \pm d.p.). As variáveis foram avaliadas quanto à normalidade de distribuição, usando o teste Kolmogorov-Smirnov. Para os dados com distribuição normal as análises foram conduzidas por meio da ANOVA (SAS, 2000). As comparações entre médias foram feitas pelo teste de Tukey. Os testes estatísticos usaram probabilidade de $5 \%$.

ganho na cadência ao trote $(\mathrm{P}>0,05)$. Contudo, foi observado melhora nos ganhos da regularidade e simetria $(\mathrm{P}<0,05)$, em que os melhores resultados foram encontrados aos cavalos submetidos a ginástica laboral realizada de três à quatro vezes/semana.

Tabela 1. Valores médios com desvio padrão $( \pm)$ dos ganhos das variáveis biomecânicas ao trote de cavalos terapeutas submetidos a frequências semanais crescentes de execução da ginástica laboral

\begin{tabular}{l|c|c|c|c|cc}
\hline \multirow{2}{*}{ Variável Biomecânica } & \multicolumn{5}{|c}{ Frequência semanal de execução da ginástica laboral } & \multirow{2}{*}{ Valor de P } \\
\cline { 2 - 6 } & $0 \mathrm{x} / \mathrm{sem}$. & $1 \mathrm{x} / \mathrm{sem}$. & $2 \mathrm{x} / \mathrm{sem}$. & $3 \mathrm{x} / \mathrm{sem}$. & $4 \mathrm{x} / \mathrm{sem}$. & \\
\hline G-CAD $^{1}$ & $-0,1 \pm 0,02$ & $-0,1 \pm 0,02$ & $-0,1 \pm 0,03$ & $0,1 \pm 0,01$ & $0,1 \pm 0,01$ & 0,0622 \\
(passada/seg) $_{\text {G-REG }^{2}(/ 200)}$ & $-51,9 \pm 2,61^{\mathrm{d}}$ & $-31,0 \pm 5,31^{\mathrm{c}}$ & $12,0 \pm 5,53^{\mathrm{b}}$ & $15,4 \pm 4,09^{\mathrm{a}}$ & $16,0 \pm 4,25^{\mathrm{a}}$ & 0,0001 \\
G-SIM $^{3}(\%)$ & $-45,7 \pm 5,75^{\mathrm{d}}$ & $-17,9 \pm 1,77^{\mathrm{c}}$ & $19,8 \pm 4,22^{\mathrm{a}}$ & $14,9 \pm 3,01^{\text {ab }}$ & $20,6 \pm 5,66^{\mathrm{a}}$ & 0,0060 \\
\hline
\end{tabular}

${ }^{1} \mathrm{G}-\mathrm{CAD}=$ ganho na cadência; ${ }^{2} \mathrm{G}-\mathrm{REG}=$ ganho na regularidade; ${ }^{3} \mathrm{G}-\mathrm{SIM}=$ ganho na simetria. ${ }^{a, b, c, d}$ Médias com letras diferentes na linha, diferem entre si pelo de Tukey.

O principal objetivo do treinamento, em relação ao movimento tridimensional do trote de equinos, é promover a coordenação, equilíbrio e propulsão durante deslocamento (BIAU \& BARREY, 2004b). Assim, espera-se que um bom trote possua boa regularidade e simetria, realizados com elasticidade, mantendo-se a frequência de passada (BURLA et al., 2014). Os resultados mostrados na tabela 1 apresentam-se concordantes a estes propósitos, ou seja, manutenção da frequência da passada com incrementos na regularidade e simetria nos cavalos submetidos a ginástica laboral. OLIVEIRA et al. (2015), também observaram melhoras significativas na qualidade do movimento ao passo em cavalos de equoterapia submetidos a ginástica laboral realizada três vezes por semana.

Ainda, o ganho na SIM, no atual trabalho, apresentou-se como a variável biomecânica mais sensível a inclusão de rotina de exercícios laborais aos cavalos de terapia, apresentando incrementos positivos a partir da execução de duas sessões semanais. Isto pode ter ocorrido 
pela maior simetria muscular nos cavalos submetidos a ginástica laboral, resultando em ganhos na variável biomecânica SIM de 19,8 e $20,6 \%$, para realização de duas e quatro sessões semanais, respectivamente. Pesquisa realizada por STUBBS, et al. (2011), corroboram esta justificativa, em que a realização de exercícios de mobilização dinâmica em cavalos (cinco vezes/semana), promoveu aumentos na simetria da musculatura epaxial, mensurados por meio do ultrassom.

Existe um consenso em que o peso e a força muscular sofrem interferência da idade do cavalo e, assim, podem influenciar a locomoção (BIAU \& BARREY, 2004a). Em se tratando de equinos idosos, como os utilizados na presente pesquisa (média de 19 $\pm 3,5$ anos), a idade avançada torna-se em um fator negativo à qualidade do movimento tridimensional. Sendo assim, os bons resultados alcançados às variáveis biomecânicas (Tabela 1) foram oriundas da inclusão de exercícios físicos de suporte aos cavalos de equoterapia, como a ginástica laboral.

Vale ressaltar que, as variáveis

\section{CONCLUSÕES}

A realização de ginástica laboral em cavalos terapeutas, a partir de três sessões semanais, melhora o movimento

\section{AGRADECIMENTOS}

Agradece-se ao suporte financeiro à pesquisa concedido pela Fundação de

\section{REFERÊNCIAS}

BARREY, E., GALLOUX, P. Analysis of the equine jumping technique by accelerometry. Equine Veterinary Journal, v.23 (Suppl.), p.45-49, 1997.

BARREY, E.; DESLIENS, F.; POIREL, D.; BIAU, S. Early evaluation of dressage ability in different breeds. Equine Veterinary Journal, v. 34(Suppl.), p. 319-
REG e SIM, além de qualificarem o movimento tridimensional do trote, são utilizadas como índices para identificar a ocorrência de claudicação nos cavalos em suas diversas modalidades esportivas (BARREY et al., 2002). Neste sentido, LOPEZ-SANROMÁN et al. (2012), verificaram redução de $50 \%$ no valor da REG em cavalos sedados com xilazina e monitorados biomecanicamente por meio do acelerômetro. O incremento no ganho destas variáveis observados na tabela 1 , em cavalos submetidos a ginástica laboral, a partir da frequência de duas sessões semanais, expressam o melhor deslocamento destes animais ao trote, de forma equilibrada e, consequentemente, sem claudicação. Esta resposta é de grande relevância, pois a claudicação estabelecida, mesmo quando não visível, interfere negativamente no bem-estar dos animais, ou seja, diminuindo a qualidade de vida dos equinos. Desta forma, a inclusão da ginástica laboral, por no mínimo duas vezes/semana, atua como ferramenta de prevenção à incidência de lesões que causam claudicação nos cavalos terapeutas.

tridimensional do trote, devido ao ganho na regularidade e simetria, mantendo-se a mesma cadência de passada.

Amparo à Pesquisa do Estado de São Paulo, FAPESP 2017/04458-2.

\section{4, 2002.}

BIAU, S.; BARREY, E. Relationship between stride characteristics and score in dressage tests. Pferdeheikunde, v.20, p. 14, 2004b.

BIAU, S.; BARREY, E. The trot characteristics during the first years of dressage training. Pferdeheikunde, v.20, 
p. 1-4, 2004a.

BURLA. J. B.; OSTERTAG. A.; WESTERATH. H. S; HILLMANN. E. Gait determination and activity measurement in horses using an Accelerometer. Computers and Electronics in Agriculture, v. 102, p.127133, 2014.

ENCHEFF, J.L., C. ARMSTRONG, M. MATERSON, C. FOX, P. GRIBBLE. Hippotherapy effects on trunk, pelvic and hip motion during ambulation in children with neurological impairments. Pediatric Physical Therapy, v. 24, p. 242-250, 2012.

HAKANSON, M., MÖLLER, M., LINDSTRÖM, I., MATTSSON, B. The horse as the healer - A study in patients with back pain. Journal of Bodywork and Movement Therapies, v.13, p.43-52, 2009.

HIGGINS, G. How your horse moves. 1. ed. Cincinnati: David \& Charles Book. 153p, 2009.

JANURA, M.; DVORAKOVA, T.; PEHAM, C.; SVOBODA, Z. The influence of walking speed on equine back motion in relation to hippotherapy. Veterinary Medicine Austria, v. 97, p. 15, 2010.

$\begin{array}{lr}\text { LÓPEZ-SANROMÁN } & \text { A. F.J; } \\ \text { HOLMBAK-PETERSEN } & \text { R.A, }\end{array}$
SANTIAGO I.A; GÓMEZ S.A, BARREY, E. Gait analysis using 3D accelerometry in horses sedated with xylazine. The Veterinary Journal, v. 193, p. 212-216, 2012.

OLIVEIRA, K., SOUTELLO, R.V.G., FONSECA, R.,COSTA C., MEIRELLES, P.R.L.,FACHIOLLI, D.F., CLAYTON, H.M. Gymnastic Training and Dynamic Mobilization Exercises Improve Stride Quality and Increase Epaxial Muscle Size in Therapy Horses. Journal of Equine Veterinary Science, v.35, n.1112, p. 888-893, 2015.

ROSA, L.R. Análise biomecânica de um cavalo de terapia: a interferência do peso corporal e da simetria postural do praticante na qualidade do passo do cavalo. In... XII Congresso Brasileiro de Equoterapia. Brasília, p.218-229, 2006.

SILVA, M.N.G. Levantamento das afecções ocorridas nos cavalos utilizados em equoterapia no período de 2006 a 2010 em Uruguaiana-RS. Revista Brasileira de Ciência Veterinária, v.19, n.3, p.139-143, 2012.

STATISTICAL ANALYSIS SYSTEM SAS. SAS user's: guide statistics. Cary: 211p, 2000

STUBBS, N.C., KAISER, L.J., HAUPTMAN, J., CLAYTON, H.M. Dynamic mobilization exercise increase cross sectional area of musculus multifidus. Equine Veterinay Journal, v. 43, n.5, p.522-529, 2011.

STUBBS, N.C.; CLAYTON, H. M. Activate your horses core. Mason: Sport Horse Publications, 2008. 32p.

SVOBODA, Z., DVOŘÁKOVÁ, T., JANURA, M. Does the rider influence the horses movement in hippotherapy? Acta University Palacki. Olomuc. Gymn., v.41, n.4, p.37-41, 2011. 